\title{
Assessment of the Impact of the Rigidity of Nodes on the Operating Reliability of EPSs
}

\author{
Nailya Chemborisova ${ }^{1^{*}}$ \\ ${ }^{1}$ National Research University Moscow Power Engineering Institute, Moscow, Russia
}

\begin{abstract}
The operating reliability of electrical power systems is mostly based on the topology and parameters of the schemes considered in this article. The preliminary (before the calculation of operating conditions) analysis of the rigidity or sensitivity of nodes, based on the matrix of node conductivity, allows for evaluating and increasing the controllability of conditions by voltages and limiting short-circuit currents, making the reduction of active power losses in the grid more efficient. A conclusion was made regarding the consistent effects of changes in the node rigidity on increasing the EPS operating reliability.
\end{abstract}

\section{Introduction}

In the operation and development of complex electrical power systems (EPSs), one of the illustrative examples being metropolis power supply systems (MPSSs), it is becoming more and more difficult to enhance reliability, improve control conditions and ensure resilience. This is partially related to the following factors: an increased number of interrelated energy facilities; the growing maximum power of electrical systems; complicated grid layouts; the improved coherence of individual elements of the system; complications in ensuring the required controllability levels of facilities; and increased levels of short-circuit (SC) currents.

It should be noted that several factors must be taken into account when designing MPSSs. Furthermore, there are a plethora of grid layout configurations that have various capabilities, properties, and indicators defining various levels of reliability. The scheme presented in [1] is deemed to be a near-optimal representation of a metropolis's power supply. However, in due to less than ideal urban planning and building construction, in practice it is impossible to use all recommended principles of optimal electrical grid organization, which entails reduced operating reliability of power systems and of power supply to consumers. Therefore, given a pre-determined set of common properties, a structural, grid-specific preliminary analysis is required. This analysis will allow for the design of future similar grids, and will correct the errant properties of existing grids.

Based on the specifics of metropolis development, some accompanying unresolved challenges can be considered [2]:

1. Improving the reliability of power supply to consumers;

2. Re-distribution of power exchanges and reduction of loading on over-loaded grid elements (OHLs, transformers);
3. Improving the capacity of system-forming and distribution grids of metropolises;

4. Improving the breaking capacity of $110 \mathrm{kV}+$ switches in order to eliminate the need for sectioning in grids.

To keep up with these challenges, control must be optimized and grid capabilities must be used more efficiently. A one-linear metropolis model can serve as an appropriate basis for the preliminary assessment of MPSSs functioning in various conditions, to arrange a fault-current limiting device (FCLD) or other control devices. A typical example of an MPSS is the Moscow Power System, a part of the Central Integrated Power System providing power to Moscow and the Moscow Region.

\section{Substantiation of the Method for Analyzing Modes and Their Reliability}

One of the EPS properties that must be accounted for is the rigidity of its nodes. For a specific point, it is characterized by loading growth for which the voltage level and phase change by a unit: the higher the growth, the more the EPS rigidity in this point [3] and the less the response behavior (sensitivity).

For the quantitative assessment of rigidity and response behavior, a node sensitivity factor can be used $[4,5]$. It shows the extent of voltage change in the node relative to the nominal voltage when the loading increased, in the considered case, by $1 \mathrm{MW}$ and 1 MVAr [8].

$$
k_{s}=\frac{U_{0}-U_{i}}{U_{\text {nom }} \cdot S_{\text {in }}} \cdot 100 \%
$$

Here, $U_{\mathrm{O}}$ is the initial voltage in the node before loading increased, $U_{\mathrm{i}}$ is the initial voltage in the node after loading increased, with the difference between

\footnotetext{
* Corresponding author: nelya1998@mail.ru
} 
them in the numerator $\Delta U, U_{\text {nom }}$ is the nominal voltage in the node, $S_{\text {in }}$ is the loading increase increment in terms of power.

In rigid nodes, the sensitivity factor will be less than in the sensitive ones. Using this factor, one can evaluate the sensitivity of the considered node to changes in the loading parameters. There are sensitive groups of elements, the loading parameters of which significantly vary due to random changes in the layout or conditions, and rigid groups of elements, the loading parameters of which almost do not vary due to external action [3]. It should be noted that when using a more precise method for searching sensitive nodes, as suggested in [6], one needs the results of singular or spectral analysis of the Jacobian matrix (written in power imbalances in the polar coordinate system) used in calculations of steadystate conditions (that do not always give a solution, even if it exists). The analysis of the properties of electrical power systems is related to the issue of marginally sensitive and rigid nodes caused by the irregularity of the EPS structure.

In designing and operating EPSs with a larger number of grid elements, it is rather hard to calculate and analyze electrical conditions without using a systemic approach. Furthermore, the cost-effectiveness of the current conditions can be decreased; the total losses of active power in the grid $\Delta P_{t}$ can rise due to the irregular areas of schemes that usually impair power system conditions. The control solution can be simplified by preliminarily analyzing the properties of the EPS substitution scheme and its parameters.

EPS rigidity primarily defines a high-voltage grid. It must be sufficiently developed, redundant and connect the primary parts of the power systems and nodes with high loading. Closed schemes of electrical grids having several power supplies are the most reliable ones. However, short-circuit currents (SCCs) grow in closed grids, which reduce reliability and become dangerous for switches and equipment installed during previous periods of EPS development [7]. To limit SC currents, current-limiting reactors can be installed and the grid can be sectioned; when sectioning, SC currents are reduced, but the electrical bond between the sectioned parts of the grid is impaired, which may result in the reduced reliability of power supply to consumers. Sectioning disadvantages also include reduced rigidity of the scheme; worsened normal and post-emergency conditions; complicated voltage control; increased power and energy losses in the grid, so sectioning is considered as a forced temporary measure.

In this manner, the goal of increasing EPS controllability has contradictory requirements. On the one hand, to enhance reliability, it is necessary to increase the scheme rigidity, while on the other hand, it is required to reduce it to limit SC currents that also affect reliability. One of the options is using compensators in sensor nodes and SCC limiters (reactors, sectioning) in the most rigid nodes that can be considered as control nodes. While this results in somewhat reduced sensitivity and rigidity of marginal nodes, increased regularity of the system, it also leads to the limitation of short-circuit currents.
However, another issue arises when reducing the rigidity of nodes. The voltage levels and capacity of system-forming and distribution grids start going down [8], which influences the performance reliability.

Since real EPSs require a system to control their steady-state and emergency conditions, it is required to preliminarily (before calculating the steady-state conditions or short-circuit currents) analyze the grid structure, its layout and operating parameters in order to have a benchmarking of the extent of changes in operating parameters in various nodes.

\section{Method of Balance Conductivities}

When forming the matrix of nodal conductivities $[Y]$ necessary to solve the equations of the steady-state condition [7], one can acquire additional information on nodes based on the differences $\Delta Y$ (balance conductivities) between the inherent conductivity in each node $Y_{i i}$ and the sum of its mutual conductivities $Y_{i j}$. The elements comprising the matrix $[\Delta Y]$ must be considered on a complex plane when ranking the acquired results in the descending (ascending) order. The nodes where the maximum values of the reactive components $[\Delta Y]+j \Delta B$, $-j \Delta B$ are obtained require increased attention when arranging control, during calculations and the analysis of conditions. For example, when controlling the conditions, one of the tasks is to find the place of inputting the most efficient control action (CA) to change voltages and consumption of reactive power in the electrical system, and to reduce losses of active power in the grid. If the resulting difference $j \Delta B$ has a capacitive nature, the voltage in the node is maintained rather efficiently. The nodes with the highest capacitive difference will be the most rigid as compared with other nodes. If $\Delta B$ has the highest values in modulus and an inductive nature, voltage will be maintained less efficiently than in a rigid node, since this node will consume additional reactive power, and the node is considered to be sensitive [8]. When comparing two nodes with an inductive difference of conductivities, the voltage in the node is better maintained with a less difference in modulus. Then one can rank the nodes by the extent of changes in the difference of conductivities and acquire a list of nodes ranked by the level of rigidity. This preliminary analysis of the grid parameters identifies rigid and sensitive nodes and allows for the rational arrangement of reactive power compensators in the grid or SCC limiters.

Using a rather simple analysis, one can find problematic nodes in a formalized manner (with the highest SCC values, with the highest changes of voltage in the node, etc.). This analysis requires no timeconsuming calculations and information which is rather conventional for calculations of steady-state conditions. It allows developing a systemic approach to the condition control, making it the most efficient, which is especially urgent in existing grids of metropolises.

It is known that, in a regular grid, the natural distribution of powers is also economical [1,9], and 
close electrical grids are usually irregular (with various $\mathrm{X} / \mathrm{R}$ ratios in various sites). The highest irregularity is found in outlines containing various nominal voltages, transformers, which results in changed power exchanges by branches and voltage in nodes, with power losses increased.

In these conditions, to compare schemes by the assessment results of some quality indicators of supplied power energy, voltages in nodes and losses in the grid, a sufficiently simple approach will be required, which allows taking into account the specifics of operation of complex irregular systems.

A comparison of several schemes by voltage levels in nodes may seem necessary in designing EPSs. A scheme with a large number of sensitive nodes will have reduced voltages as compared with the voltages in the scheme with more rigid nodes, but also decreased SCCs [10]. The power system can be considered as several regions with prevailing nodes having specific properties (sensitive or rigid).

\section{Calculation Results for Increasing Reliability}

The analysis of changes in operating parameters is interesting first of all in the most sensitive nodes, since they respond better during changes. Rigid nodes are almost of no interest in voltage normalization, since volt-age levels in them are only slightly changed in the case of similar impacts.

To demonstrate the capabilities of such an approach, a famous testing scheme was considered as given in Fig. 1, as well as a modified annular scheme based on it (Fig. 2).

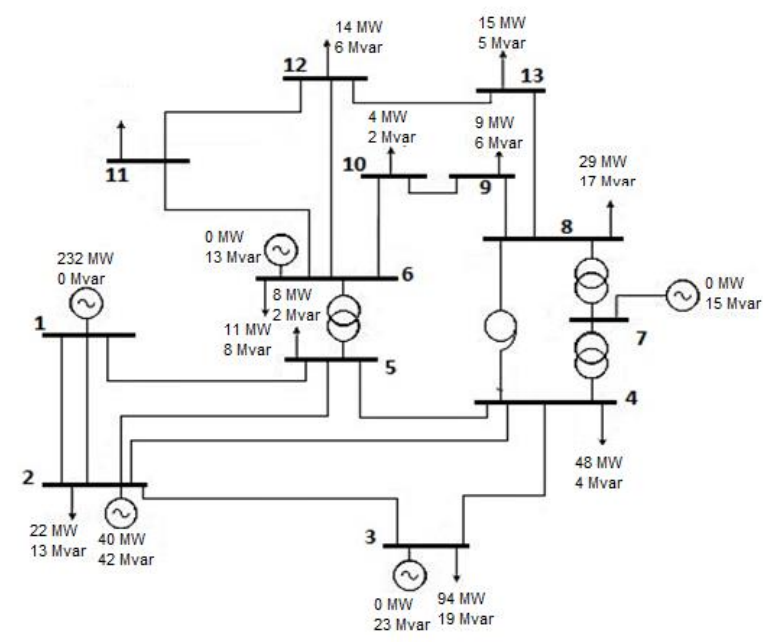

Fig. 1. Option 1 - Test scheme (complex closed).

Table 1 gives the calculation results for the most rigid (6) and sensitive (11) nodes $110 \mathrm{kV}$ for sensitivity factors and SCC (three-phase SC).

To conveniently compare the options, currents are given in relative units with regard to the maximum current in the most rigid node (6) for option 1 (complex closed scheme). ks are calculated using the above formula.

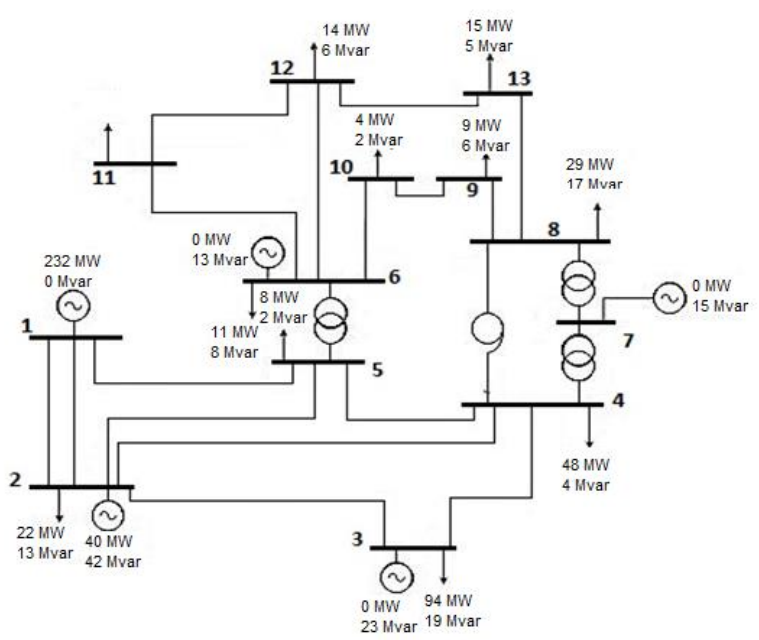

Fig. 2. Option 2 - Modified scheme (annular).

Table 1. Results of comparable calculations of $k_{\mathrm{s}}$ and SCC.

\begin{tabular}{|c|c|c|c|}
\hline $\begin{array}{c}\text { Scheme } \\
\text { No. }\end{array}$ & Node no./type & $k_{\mathrm{s}}$ & $\mathrm{I}_{3 \mathrm{ph}} /$ r.u. \\
\hline 1 & $6 /$ rigid & 0.118 & 1.000 \\
\hline 1 & $11 /$ sensor & 0.132 & 0.853 \\
\hline 2 & $6 /$ rigid & 0.188 & 0.905 \\
\hline 2 & $11 /$ sensor & 0.214 & 0.743 \\
\hline
\end{tabular}

It should be noted that the numbers of the most rigid and sensitive nodes acquired using the matrix of nodal conductivities have fully coincided with the calculation results of worsening, e.g., the capabilities of various schemes in maintaining voltage can be compared using the minimal values of balance conductivities: the higher the balance conductivity by modulus in the most sensitive node, the higher the predicted voltage change (the sensitivity factor), but the lower SCC. If it is necessary to maintain voltage in the sensitive node for two schemes with various values of such balance conductivities, the preferable scheme is the one with a lower value in modulus, since the voltage will be better maintained. For example, in sensitive node 11, the sensitivity factor is higher in option 2 , so the voltage in the most sensitive node will be maintained less efficiently. This approach allows for the choosing of nodes, the voltage control of which should receive special attention, and comparing schemes by their capability to maintain voltage in the most sensitive nodes.

If a scheme with the minimal SCC is required, the comparison of the most rigid nodes will be the most informative. Balance conductivities lower in modulus in the most rigid node will characterize lower SCC. Since the nodes were ranked using the balance conductivity method before calculations of the steady-state conditions and SCC, followed by the calculation of worsening for 
the selected nodes and of the sensitivity factor that also allowed finding rigid and sensitivity nodes in the options ( $k_{\mathrm{s}}$ in rigid nodes is lower than in the sensitive ones, as per the definition of rigid nodes), the using of this method can be deemed correct in the preliminary evaluation of the rigidity of nodes. When selecting a scheme with the lowest SCC, the best option in rigidity evaluation is option 2, since for rigid node 6 , the sensitivity factor is higher, e.g., the node is less rigid than in option 1. When comparing after SCC calculation using the option having lower SCC values, option 2 is also the choice. It should be noted that SCC values for the considered options are higher in rigid nodes than in the sensitive ones. This proves the opportunity to use the method of balance conductivities for preliminary benchmarking of nodes in SCC under a single option and to compare schemes by relative values of SCC in various options.

To confirm the conclusions made, a retrospective scheme of the Moscow Energy System was considered, with a similar analysis performed. The comparison revealed that the most rigid node maintains the voltage very well, since $\Delta U$ is below $1 \mathrm{kV}$, and $\Delta U$ in the most sensitive node during voltage changes is within $(+8.5)-(-$ 5.7) $\mathrm{kV}$ relative to the nominal level. It should be noted that these nodes have approximately equal basic load and function at the same level of voltage $(110 \mathrm{kV})$ in annular grids. Then, in the grids typical of annular schemes, this approach will operate very efficiently. When comparing SCCs and places of the node in the ranked list of $100 \mathrm{kV}$ nodes, the following current values have been obtained: Butyrki (most rigid) - $114.3 \mathrm{kA}$, Ochakovo (least rigid) - 77.1 kA, which is significantly higher than the breaking capacity of switches at this voltage level. For sensitive nodes $100 \mathrm{kV}$ located on the opposite end of the ranked list of nodes, these values are significantly lower, for example, Beskudnikovo $31.2 \mathrm{kA}$, Vladimir (most sensitive) - 28.1 kA.

The method of balance conductivities resulting in the ranked list of nodes as suggested herein allows for significant substantiation of promising schemes in designing or repair schemes in operation, finding possible options of the grid scheme that ensure grid controllability and power supply reliability of its individual nodes.

\section{Conclusion}

The goal of increasing EPS controllability has contradictory requirements. On the one hand, to enhance reliability, it is necessary to increase the scheme rigidity, while on the other hand, it is required to reduce it to limit SC currents, which also affect reliability. To assess the potential for increasing the controllability of the electrical grid (by maintaining voltage in the most sensitive nodes and reducing SCCs in the most rigid nodes) and improving the reliability of individual nodes, a preliminary analysis is required to form a ranked list of nodes based on the calculation of balance conductivities. This analysis should precede the calculation of steady- state conditions and SCCs and should use elements of the node admittance matrix. The same approach allows benchmarking various configurations of grid schemes to find options having enhanced grid controllability and power supply reliability of individual nodes, and it can also substantially reduce the number of considered scheme options during design and operation.

The opportunities for using the suggested approach were checked by calculations for the known test scheme and the Moscow Energy System, which proved it to be correct.

\section{References}

1. D.P. Faibisovich (Ed.), Guidebook for designing electrical grids, 2nd ed. (ENAS, Moscow, 2012)

2. V.V. Sergeev, V.N. Savinov, V.S. Pavlikov, Electrical Stations, 11, 59-67 (2007)

3. N.E. Savoskin, Reliability of electrical systems (Publishing House of the Penza State University, Penza, 2004)

4. S. Conti, S. Raiti, G. Vagliasindi, Industrial Electronics (ISIE), 2010 IEEE International Symposium on, 2548-2554 (IEEE, 2010)

5. Q. Zhou, J. Bialek, Proc. of the 16th Power Systems Computation Conference (PSCC2008), Glasgow, Scotland (2008).

6. O.N. Voitov, N.I. Voropai, A.Z. Gamm, I.I. Golub, D.N. Efimov, Irregularities analysis of electrical power systems (Nauka, Sibirskaya izdatelskaya firma RAN, Novosibirsk, 1999)

7. V.I. Idelchik, Electrical systems and grids: Study book for high schools (Energoatomizdat, Moscow, 1989)

8. O.V. Frolov, N.Sh. Chemborisova, Energy industry: Control, quality, and efficiency of using energy resources: Collection of works of the Sixth AllRussian Scientific Conference with International Participants, 1, 100-104 (AmGU Publishing House, Blagoveshensk, 2011)

9. O.A. Lopatin, N.Sh. Chemborisova, Electricity, 3, 10-12 (2011)

10. O.V. Frolov, N.Sh. Chemborisova, New in the Russian Electrical Power-Engineering, 4, 33-40 (2011) 